\title{
Zielperspektiven in die Coaching-Praxis integrieren - Ein integratives Modell zielorientierten Coachings
}

\author{
Anthony Grant ${ }^{1}$ \\ Online publiziert: 22. Dezember 2017 \\ (c) Der/die Autor(en) 2017. Dieser Artikel ist eine Open-Access-Publikation.
}

\section{Zusammenfassung}

Im Artikel werden bekannte Theorien zur Zielfindung, -formulierung und -umsetzung beschrieben. Aus den beschriebenen Elementen wird ein integratives Modell zielorientierten Coachings entwickelt. Das Modell kann Coaches dabei unterstützen, ihren Coachees im Zielfindungs- und Umsetzungsprozess zu helfen. Ziele werden dabei nicht als monolithisches Konstrukt, sondern als facettenreiches Element in Coachingprozess betrachtet. Das Modell kann daher nicht Schritt für Schritt abgearbeitet werden. Der Schlüssel liegt eher in einer sachkundigen und flexiblen Handhabung des Modells durch den Coach. Das Verständnis verschiedener Zielarten und ihrer Bedeutung für Veränderung, sowie das Begleiten von Zielfindungsprozessen, ermöglicht es professionellen Coaches effektiv mit ihren KlientInnen an Einsichten und Verhaltensveränderungen zu arbeiten die ihre Arbeitsleistung, ihr Arbeitserleben, sowie - und dies ist der wichtigste Punkt - ihr persönliches Wohlbefinden und Selbstverständnis erhöhen.

Schlüsselwörter Zieltheorie $\cdot$ Zielfindung $\cdot$ Zielformulierung $\cdot$ Zielumsetzung

\section{Integrating Perspectives on Goals in Coaching Practice. An Integrated Model of Goal-focused Coaching}

\begin{abstract}
This article describes well-known theories on goal alignment, goal articulation and goal implementation. Based on the described elements, an integrative model of goal-oriented coaching is developed. The model can support coaches to help their clients in aligning and implementing goals. Goals are thereby not considered as monolithic constructs, but as multi-faceted elements of a coaching process. Thus, the model cannot be executed step-by-step. The key to success lies more in a competent and flexible handling of the model by the coach. By understanding the different types of goals and their relationship to the process of change, and through facilitating the goal alignment process, professional coaches can work more efficiently with their clients, helping them to achieve insight and behavioral change that enhances their workplace performance, their professional working lives and, most importantly, their own personal well-being and sense of self.
\end{abstract}

Keywords Goal theory $\cdot$ Goal alignment $\cdot$ Goal articulation $\cdot$ Goal implementation

Dieser Beitrag ist die deutsche Übersetzung des Buchkapitels „New perspectives on goal setting in coaching practice: an integrated model of goal-focused coaching" von A. Grant (2013b) aus dem Buch "Beyond goals: effective strategies for coaching and mentoring", herausgegeben von D. Clutterbuck, D. Megginson und S. David, erschienen bei Gower Publishing.

Anthony Grant

anthony.grant@sydney.edu.au

1 Coaching Psychology Unit, The University of Sydney NSW 2006, Sydney, Australien
Seltsamerweise wird die Verwendung von Zielen im Coaching kontrovers diskutiert. Jene, die sich gegen das Setzen von Zielen im Coaching aussprechen, argumentieren, dass es Coaching-Gespräche begrenzen und das Aufkommen anderer Fragen behindern würde, oder dass Zielsetzungen sich in der Regel auf Themen beziehen, die zwar leicht gemessen werden können, aber keine wirkliche Bedeutung haben. Andere sagen, das Setzen von Zielen führe zu häufig da$\mathrm{zu}$, dass Coaches ihre KlientInnen in Richtung eines vorher vereinbarten, jedoch unpassenden Ziels drängen, und dass 
dieser Ansatz in ,faulem“, mechanischem Coaching resultiere. Manche Coaches behaupten gar, dass sie im Coaching nie Ziele verwenden. Stattdessen würden sie ihren KlientInnen helfen, ihre Werte zu entdecken und ihre Intentionen zu klären, um dann mit ihnen an der Erfüllung ihrer Wünsche zu arbeiten. Wieder andere Coaches sprechen davon, dass sie ihren KlientInnen behilflich seien, die persönliche Geschichte neu zu schreiben, den Kompass neu auszurichten, sich sicher durch die Wirren des Lebens zu bewegen, oder Entwicklung und Transformation zu beflügeln.

Das Setzen von Zielen hat mittlerweile sogar in manchen Bereichen der akademischen Psychologie einen schlechten Ruf. AutorInnen diskutieren das Setzen von Zielen sei außer Kontrolle geraten und beklagen eine vermeintliche Überverwendung von Zielen in Organisationen (Ordóñez et al. 2009). Auch in der Coaching-Literatur werden die Grenzen von Zielsetzungen diskutiert (Clutterbuck 2008, 2010).

All diese Punkte sind ernst zu nehmen. Trotzdem bin ich der Meinung, dass die Zieltheorie der Coaching-Forschung und -Praxis viel zu bieten hat. Es gibt umfangreiche und über viele Jahre gewachsene Forschung zu Zielen und Zielsetzungen. Eine Suche in der Datenbank PsycINFO im April 2015 mit dem Keyword „Ziele“ (,goals“) erzielte über 73.118 Treffer. Die wissenschaftliche Literatur zur Verwendung von Zielen im Führungskräftecoaching fällt jedoch deutlich kleiner aus, und ergab bei den Keywords „Ziele“ (,goals“) und „Führungskräftecoaching“ (,executive coaching") gerade mal 52 Treffer. Die meisten Studien befassen sich mit der unterschiedlichen Verwendung von Zielen im Führungskräftecoaching (z. B. Bono et al. 2009; Cowan 2013; Gregory und Levy 2015; Lewis-Duarte 2010; McKenna und Davis 2009b; Stern 2009), während nur ein paar empirische Arbeiten untersuchen, wie Führungskräftecoaching die Zielerreichung beeinflusst (z. B. Benavides 2009; Burke und Linley 2007; Freedman und Perry 2010; Grant et al. 2009; Milare und Yoshida 2009; Schnell 2005; Smither et al. 2003; Turner 2004). Erstaunlich wenig Artikel diskutieren theoretische Bezüge, die die Zieltheorie explizit mit Führungskräftecoaching verbinden. Zum Beispiel zeigen sowohl Gregory und Levy (2015) als auch Gregory et al. (2011) auf, dass die Kontrolltheorie (in welcher Ziele und Feedback zwei entscheidende Elemente sind) einen wichtigen Bezugsrahmen für Coaching darstellen kann, und Grant (2006) beschreibt einen integrativen, zielorientierten Ansatz im Führungskräftecoaching.

Der Artikel beruht auf verschiedenen englischen Beiträgen und ergänzt frühere Publikationen (Grant 2002, 2006, 2013a, b). Er stützt sich auf die Zielsetzungs-, Selbstbestimmungs- und Persönlichkeitsliteratur der Verhaltenswissenschaften, diskutiert das Konzept ,Ziele‘, stellt eine für die Coaching-Praxis taugliche Zieldefinition vor und beschreibt ein Modell zielorientierten Coachings, das eine Reihe unterschiedlicher Perspektiven auf die Zieltheorie in- tegriert und diese Erkenntnisse im Coaching-Kontext greifund anwendbar macht. Außerdem wird ein Forschungsprojekt vorgestellt, das den Zielorientierungs-Fähigkeiten eines Coaches in Bezug auf erfolgreiche Resultate im Coaching eine zentrale Rolle zuschreibt.

\section{Unterstützen SMARTe Ziele unsauberes Denken im Coaching?}

Ziele und Zielkonstrukte wurden in der akademischen Psychologie umfassend diskutiert und erforscht (Moskowitz und Grant 2009) und in der einschlägigen Literatur entwickelte sich ein differenziertes Verständnis von Zielen und ihrer Bedeutung für das menschliche Verhalten. Dies gilt jedoch nicht für die Coaching-Literatur. Bei einem Überblick über diese wird deutlich, dass das Verständnis vieler Coaches von Zielen sich auf Akronyme wie SMART (ursprünglich beschrieben von Doran 1981; Raia 1965) beschränkt ein Akronym, das Ziele mit spezifischen, mess- und erreichbaren, relevanten und zeitgebundenen Handlungsplänen gleichsetzt (Hinweis: die genaue Auslegung des Akronyms unterscheidet sich je nach AutorIn).

Während die im SMART-Akronym enthaltenen Konzepte tatsächlich weitgehend von der Zieltheorie gestützt werden (z. B. Locke 1996), und sich in einigen CoachingSituationen als nützlich erweisen können, bin ich der Meinung, dass die weitverbreitete Meinung Ziele seien dasselbe wie SMARTe Handlungspläne die Entwicklung eines differenzierten Verständnisses und Gebrauchs der Zieltheorie im Coaching erschwert hat. Es ist wichtig festzuhalten, dass Akronyme wie SMART zwar durchaus nützliche Gedächtnisstützen darstellen - leicht erinnerbare Orientierungspunkte für komplexe Zusammenhänge. Allerdings kann die Anwendung solcher Gedächtnisstützen ohne klares Verständnis der zugrundeliegenden Zusammenhänge leicht zu unsauberem Denken führen: zu unsachlichen Entscheidungen und der Verbreitung einer falsch verstandenen und mythenhaften Lehrmeinung, die es für PraktikerInnen schwermacht, sich mit den zugrundeliegenden Zusammenhängen auseinander zu setzen. Ich hoffe, dass dieser Artikel die Thematik voranbringen wird.

Was sind Ziele? Wenn dieser Artikel einen bedeutsamen Beitrag in Bezug auf eine differenziertere Verwendung von Zielen und der Zieltheorie im Coaching leisten soll, brauchen wir zunächst ein klares Verständnis davon, was wir unter Zielen verstehen. Der Begriff „Ziel“ wird gemeinhin verstanden als „,der Zweck, auf den ein Bemühen gerichtet ist; ein Richtwert oder Ergebnis" (siehe z. B. www. thefreedictionary.com). Während solche Definitionen im Alltag ausreichend sind, brauchen wir im Coaching ein genaueres Verständnis des Konstrukts. Um eine differen- 
zierte Ausarbeitung des Konstrukts „Ziel“ zu ermöglichen, wurden von Forschenden über die Jahre viele weitere Begriffe vorgeschlagen, darunter „Referenzpunkte“ (,reference values“) (Carver und Scheier 1998), „Leitfäden“ (,self-guides“) (Higgins 1987), „persönliches Streben“ (,personal strivings“) (Emmons 1992) oder „persönliche Projekte“ (,personal projects“) (Little 1993). Derart weit gefasste Definitionen erlauben es jedoch nicht zwischen Zielen, Wünschen und Ergebnissen zu unterscheiden, und erfassen auch nicht die Essenz des Zielkonstrukts.

Wie zuvor bereits erwähnt, ergibt sich der Sinn von Zielen in Hinsicht auf Veränderungsprozesse, in denen Ziele eine Rolle in dem Übergang von einem Ist-Zustand zu einem erwünschten Zustand oder Ergebnis zukommt (z. B. Klinger 1975; Spence 2007). Als solches wurde das Zielkonstrukt in Bezug auf Kognitionen (Locke 2000), Verhalten (Bergh et al. 2001; Warshaw und Davis 1985) und Affekt (Pervin 1982) beschrieben (für eine weitere Betrachtung dieser Punkte siehe Street 2002). Da diese drei Bereiche für Coaching von großer Relevanz sind, bin ich der Ansicht, dass eine Definition von Zielen für das Coaching alle drei umfassen sollte.

Cochran und Tesser (1996) bieten eine verständliche Beschreibung von Zielen als ,kognitives Bild eines Ideals, das im Gedächtnis zum Vergleich mit einem tatsächlichen $\mathrm{Zu}$ stand abgespeichert ist; eine Repräsentation der Zukunft, die die Gegenwart beeinflusst; ein Wunsch (Freude und Befriedigung werden durch Zielerfolg erwartet); eine Quelle der Motivation, ein Anreiz zu handeln" (S. 100, zitiert nach Street 2002). Dieses Verständnis von Zielen könnte sich speziell für Coaching als nützlich erweisen, denn wie Street (2002) argumentiert, betont es die Bedeutung der Kognition (als kognitive Bilder), wie auch die von Affekt und Verhalten. Zusätzlich wird der Sinn eines Ziels als „Quelle von Motivation und als Anreiz" explizit benannt, was ebenfalls große Relevanz fürs Coaching besitzt.

Während diese Beschreibung einen klaren Vorteil gegenüber der Auffassung bietet, dass Ziele mit SMARTen Handlungsplänen gleichzusetzen seien, ist sie als Arbeitsdefinition allerdings etwas unhandlich. Eine prägnante Definition, die das Wesentliche beinhaltet und gleichzeitig gut anwendbar ist, stammt von Austin und Vancouver (1996). Sie beschreiben Ziele als ,interne Repräsentationen erwünschter Zustände oder Ergebnisse“ (S. 388).

\section{Ziele spielen im Coaching eine zentrale Rolle}

Definitionen von Coaching gibt es im Überfluss. Die Internationale Coaching Federation ICF definiert Coaching als „sich mit KlientInnen in einen gedankenanregenden und kreativen Prozess begeben, der diese inspiriert, ihr per- sönliches und berufliches Potenzial zu maximieren“ (ICF 2012). Die Association for Coaching definiert Coaching als ,systematischen lösungs- und ergebnisorientierten Prozess, in dem der Coach die Steigerung der Arbeitsleistung, der Lebenserfahrung, des selbstgerichteten Lernens und persönlichen Wachstums des/der Coachee unterstützt“ (AC 2012). Die World Association of Business Coaches definiert Business-Coaching als strukturiertes Gespräch mit dem Ziel, ,das Bewusstsein und Verhalten des Klienten/der Klientin zu fördern, so dass unternehmerische Ziele des Klienten/der Klientin und seiner/ihrer Organisation erreicht werden“ (WABC 2012). Der European Mentoring and Coaching Council erklärt, Coaching (und Mentoring) seien „Aktivitäten der beruflichen und persönlichen Entwicklung ..., die KlientInnen unterstützen, ... alternative Möglichkeiten in der Verbesserung ihrer Kompetenzen, Entscheidungsfindungen und Lebensqualität zu sehen und auszuprobieren, ... mit dem Ziel, dass KlientInnen ihre Leistung erhöhen und/oder sich persönlich entwickeln ...“ (EMCC 2011).

Offenbar findet sich eine hohe Übereinstimmung zwischen den professionellen Coaching-Verbänden über das Wesen von Coaching. Alle aufgeführten Definitionen von Coaching beschreiben, dass Coaching im Wesentlichen Individuen darin unterstützt, ihre intra- und interpersonellen Ressourcen zu regulieren und dahingehend auszurichten, zweckgerichtete, positive Veränderungen in ihrem Privatoder Berufsleben zu erzielen. Im Kern geht es also im Coaching darum, dass KlientInnen in ihren Selbstregulationsfähigkeiten unterstützt werden, um positive Veränderungen in ihrem Leben bewusst herbeizuführen.

\section{Selbstregulation steht im Mittelpunkt des Coaching-Prozesses}

Selbstregulation als Konstrukt wird als eine Abfolge von Prozessen verstanden, in denen das Individuum sich Ziele setzt, einen Handlungsplan entwirft, diesen in die Tat umzusetzen beginnt, die eigene Leistung beobachtet, im Vergleich zu einem Standard bewertet, und aufgrund dieser Bewertung seine Handlungen anpasst, um seine Leistung $\mathrm{zu}$ verbessern und seine Ziele besser zu erreichen (Carver und Scheier 1998). Im Coaching ist es die Aufgabe des Coaches, das Voranschreiten seines/seiner Coachee durch den Selbstregulations-Zyklus zu unterstützen. Abb. 1 zeigt ein generisches Modell der Selbstregulation.

In der Praxis lassen sich die einzelnen Schritte des Selbstregulations-Zyklus nicht scharf trennen. Jede Stufe überschneidet sich mit der folgenden. Idealerweise unterstützt ein Coaching auf jeder Stufe den Übergang auf die nächst höherliegende. Zum Beispiel sollte die Zielsetzung so vorgenommen werden, dass sich die Entwicklung und 


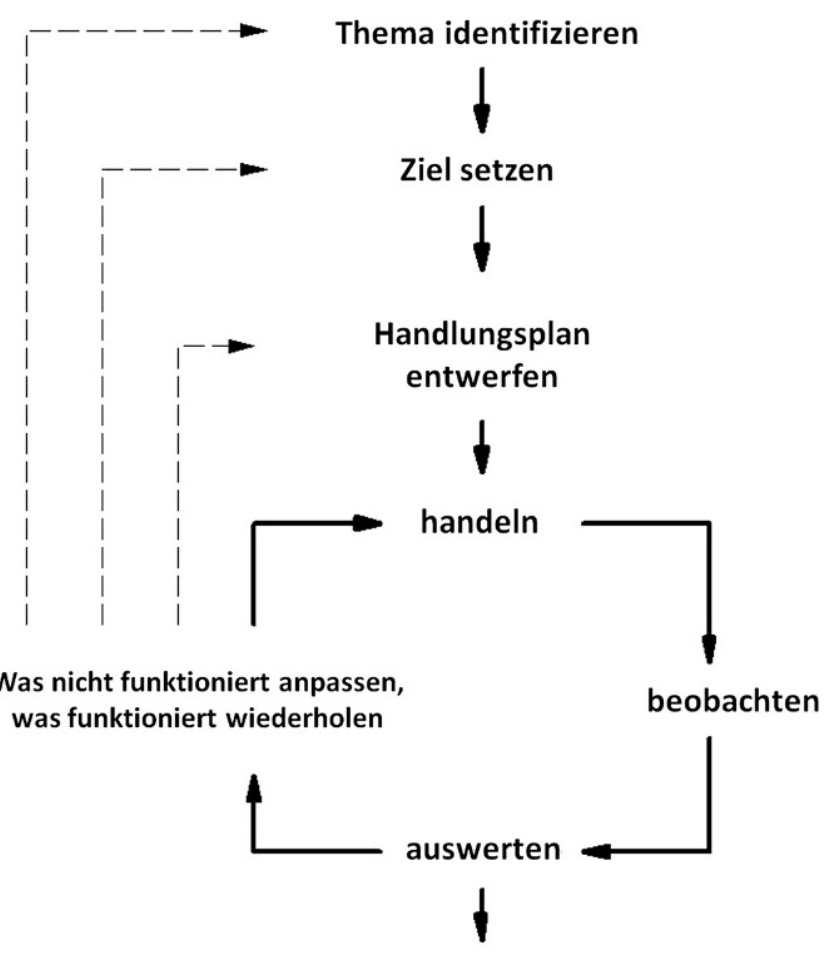

Ziel erreicht

Abb. 1 Generisches Model zielorientierter Selbstregulation

Implementierung eines Handlungsplanes daraus ergibt. Der Handlungsplan wiederum sollte das Individuum zur Handlung motivieren und gleichzeitig konkrete Punkte enthalten, wie das eigene Verhalten beobachtet und ausgewertet werden kann, so dass diese Informationen für die gemeinsame Arbeit in folgenden Coaching-Sitzungen zur Verfügung stehen.

Dieser Prozess steht eindeutig im Mittelpunkt eines Coachings. Das wird auch in den oben zitierten, durch die Berufsverbände aufgestellten Definitionen von Coaching deutlich, in denen eine aktive Orientierung hin zu einer bewusst gestalteten, positiven Veränderung ein zentraler Teil der Coaching-Gespräche darstellt. Insofern sind alle Coaching-Gespräche explizit oder implizit zielorientiert.

$\mathrm{Zu}$ wissen, wann und wie Ziele im Coaching gesetzt werden, und einzuschätzen, ob man mit KlientInnen ein explizit zielorientiertes Gespräch führen oder lieber mit abstrakten und vage formulierten Zielen arbeiten sollte, sind Fähigkeiten, die beginnende von erfahrenen Coaches unterscheiden (Grant 2011; Peterson 2011). Ein solides Verständnis des Facettenreichtums von Zielsetzungsmöglichkeiten ist also hilfreich, um den Übergang vom Anfänger bzw. Anfängerin zum Experten bzw. zur Expertin zu vollziehen. Diesem Punkt wenden wir uns nun zu.

\section{Der Facettenreichtum von Zielen}

Ziele sind kein monolithisches Konstrukt. Wenn wir Coaching durch den Blickwinkel der Zieltheorie verstehen wollen, ist es wichtig, zwischen verschiedenen Arten von Zielen zu unterscheiden. Es gibt über 20 verschiedene Arten von Zielen, die im Coaching eingesetzt werden können, zum Beispiel Ergebnisziele, distale und proximale Ziele, Annäherungs- und Vermeidungsziele, Leistungs- und Lernziele, Ziele höherer und niederer Ordnung, und die ganz konkreten Resultate, die der Coachee erreichen möchte. Da unterschiedliche Zielarten sich verschieden auf Erfolg und Erfahrung eines/einer Coachee im Prozess seines/ihres Zielstrebens auswirken, sind dies wichtige Unterscheidungen.

\section{Der zeitliche Rahmen}

Zielen einen zeitlichen Rahmen zu geben ist ein wichtiger Teil des Zielsetzungs-Prozesses, und kann die Wahrnehmung des/der Coachee auf die Erreichbarkeit des Ziels beeinflussen (Karniol und Ross 1996). Distale Ziele sind langfristige Ziele. Sie sind den Vision Statements ähnlich, über die in der Unternehmens- und Management-Literatur so gerne geschrieben wird, oder auch den ,weiten, unscharfen Visionen“" der Life-Coaching Literatur (Grant und Green 2004). Proximale Ziele sind kurzfristiger, regen eine genauere Planung an als distale Ziele (Manderlink und Harackiewicz 1984) und sind deshalb hilfreich bei der Entwicklung von Handlungsplänen. Im Grunde sind die Handlungsschritte, die üblicherweise in Coaching-Sitzungen erarbeitet werden, nichts weiter als eine Reihe von proximalen Zielen. Distale und proximale Ziele im CoachingProzess zu verbinden kann zu einer verbesserten Strategieentwicklung und erhöhtem langfristigen Erfolg führen (Weldon und Yun 2000).

\section{Ergebnisziele}

Viele Coaching-Programme konzentrieren sich explizit auf Ergebnisziele. In der Regel entsprechen diese der direkten Formulierung eines erwünschten Ergebnisses (Hudson 1999); zum Beispiel: „den Umsatz des Produkts in den nächsten 3 Monaten um $15 \%$ steigern“. Dies ist eine nutzbringende Art der Zielsetzung, denn herausfordernde, spezifisch und explizit formulierte Ergebnisziele ermöglichen einen präzisen Fokus in den Arbeitsanstrengungen, und führen bei Individuen, die über Engagement, das entsprechende Wissen und die entsprechenden Fähigkeiten verfügen, zu hoher Leistung (Locke 1996). Tatsächlich konzentrieren sich viele Coaching-Programme ausschliesslich auf die Verwendung spezifischer SMART-Ziele, und dieser Ansatz 
wird auch durch einen Teil der Literatur zu Zielsetzung unterstützt (Locke und Latham 2002).

Trotzdem können überspezifische Ergebnisziele Coachees befremden und sogar zu einem Leistungsabfall führen (Winters und Latham 1996). Bei Individuen, die in einer abwägenden oder kontemplativen Phase in ihrem Veränderungsprozess stehen (Gollwitzer 1999), kann es hilfreich sein, abstrakte oder vage Ziele zu wählen und eine weite, unscharfe Vision zu entwickeln, statt sich in Details zu vertiefen und das Aufstellen konkreter Ziele zu forcieren. Individuen an solchen Punkten des Veränderungsprozesses nehmen vage Ziele in der Regel als weniger bedrohlich und herausfordernd wahr (Dewck 1986).

\section{Annäherungs- und Vermeidungsziele}

Vermeidungsziele drücken sich als Wegbewegung von einem unerwünschten Zustand aus, zum Beispiel „Ich möchte weniger Stress bei der Arbeit haben“. Obwohl sie Ergebniszielen ähnlich sind, benennen Vermeidungsziele ihr Ziel nicht spezifisch und geben keinen Hinweis darauf, welches Verhalten zum Erreichen des Ziels günstig sein könnte. Im Gegensatz dazu drücken sich Annäherungsziele als Bewegung auf ein bestimmtes Ergebnis oder einen bestimmten Zustand hin aus, zum Beispiel „Ich möchte ein angenehmes Gleichgewicht zwischen Arbeit und Freizeit genießen“. Es überrascht nicht, dass mit Annäherungs- und Vermeidungszielen unterschiedliche Wirkungen in Zusammenhang gebracht werden. Coats et al. (1996) fanden bei Menschen mit einer Neigung zu Vermeidungszielen eine höhere Depressivität und vermindertes Wohlbefinden. Andere Studien bringen die langfristige Verfolgung von Vermeidungszielen mit geringerem Wohlbefinden in Zusammenhang (Elliot et al. 1997), und Annäherungsziele mit einer höheren akademischen Leistung und gesteigertem Wohlbefinden (Elliot und McGregor 2001).

\section{Leistungs- und Lernziele}

Leistungsziele konzentrieren sich auf eine Handlungsdurchführung. Sie vermitteln Ansporn. Das Ziel ist, bei einer bestimmten Aufgabe gut abzuschneiden, gutes Feedback von Anderen zu erhalten oder Andere zu übertrumpfen. Bei Leistungszielen konzentrieren sich die Coachees in der Regel auf ihr persönliches Können (Gresham et al. 1988). Im Führungskräfte- oder Businesscoaching könnte ein Leistungsziel lauten: „Ich möchte der beste Anwalt/die beste Anwältin auf meinem Gebiet sein.“ Leistungsziele können kraftvolle Motivatoren sein, insbesondere, wenn sich bereits früh auf dem Weg Erfolge einstellen. Allerdings können Leistungsziele Leistung auch verhindern. Dies gilt insbe- sondere dann, wenn es sich um eine sehr komplexe Aufgabe handelt oder das Ziel als sehr herausfordernd erlebt wird, und gleichzeitig die zur Verfügung stehenden Ressourcen knapp sind oder der/die Coachee ein geringes Können oder Selbstwirksamkeitsempfinden mitbringt. Zusätzlich können Leistungsziele in Situationen, in denen es um viel geht oder die von Konkurrenz geprägt sind, auch unehrliches Verhalten oder einen Unwillen zur Kooperation anregen. Die Unternehmenswelt ist voll solcher Beispiele (Midgley et al. 2001).

In vielen Fällen sind deshalb Lernziele besser dazu geeignet, Arbeitsleistungen zu erhöhen (Seijts und Latham 2001). Lernziele (manchmal auch AufgabenbeherrschungsZiele genannt) legen den Fokus auf den Lernprozess, der mit der Aufgabenbeherrschung einhergeht, statt sich auf die Aufgabe an sich zu konzentrieren. Beispiel eines Lernzieles könnte im Führungskräfte- oder Businesscoaching sein: „Ich lerne, wie ich der beste Anwalt/die beste Anwältin auf meinem Gebiet sein kann." Lernziele werden mit einer Reihe positiver Effekte in Zusammenhang gebracht, z. B., dass komplexe Aufgaben nicht als bedrohlich, sondern als positive Herausforderung erlebt werden, oder dass sie zu einem stärkeren Vertiefen in eine Arbeitsaufgabe (Deci und Ryan 2002) und zu erhöhter Gedächtnisleistung und Wohlbefinden führen (Linnenbrink et al. 1999). Wenn Teamziele als Lernziele formuliert sind, kann sich außerdem die individuelle Leistung in hochkomplexen Situationen erhöhen, und die Kooperation zwischen den Teammitgliedern gestärkt werden (Kristof-Brown \& Stevens 2001). Ein Vorteil Lernziele zu setzen ist, dass sie mit einer höheren intrinsischen Motivation assoziiert werden, was wiederum mit Leistung in Verbindung gebracht wird (Sarrazin et al. 2002).

Obwohl die unterschiedliche Formulierung dieser Zielarten scheinbar nur eine Frage der Semantik ist, spielt die Art der Zielformulierung tatsächlich eine entscheidende Rolle (Rawsthorne und Elliot 1999), und Coaches sollten diese Nuancen ernst nehmen, wenn sie mit einem zielorientierten Ansatz auf effektive Weise arbeiten wollen.

\section{Komplementäre und konkurrierende Ziele}

Außerdem sollten Coaches sich konkurrierender oder widersprüchlicher Ziele bewusst sein. Diese treten auf, wenn das Streben nach einem Ziel das Streben nach einem anderen Ziel beeinträchtigt. Manchmal sind Zielkonflikte leicht erkennbar, zum Beispiel, wenn zwei Ziele vorliegen wie: „Ich möchte mehr Zeit mit meiner Familie verbringen.“ und „Ich möchte meiner Arbeit mehr Zeit einräumen, um befördert zu werden." Ein Zielkonflikt ist aber längst nicht immer so offensichtlich. Beispielsweise könnte das Ziel „Mein Verkaufspersonal soll höhere Umsätze erzielen." in einem wahrgenommenen Konflikt mit dem Ziel „einen weniger 
autoritären Führungsstil umsetzen" stehen, wenn der/die Coachee (ein/e VerkaufsleiterIn) delegieren als schwierig empfindet und einen kontrollierenden Führungsstil im Umgang mit seinen/ihren MitarbeiterInnen gewohnt ist.

In solchen Situationen ist die Fähigkeit eines Coaches gefragt, scheinbar widersprüchliche Ziele einander anzugleichen und aus ihnen komplementäre Ziele zu entwickeln. Sheldon und Kasser (1995) legen dar, dass Kongruenz wichtig für die Zielerreichung und das Wohlbefinden ist.

\section{Unbewusste Ziele?}

Der Mensch ist ein zielorientiertes Wesen. Ohne Ziele könnten wir nicht als bewusst fühlende Wesen existieren. Carver und Scheier (1998) argumentieren, dass menschliches Verhalten ein kontinuierliches Hin- oder Wegbewegen von mentalen Zielrepräsentationen sei. Das bedeutet aber nicht, dass all diese Ziele bewusst sind. Oft verfolgen wir komplexes, ergebnisorientiertes Verhalten ohne uns bewusst Ziele gesetzt zu haben.

Zum Beispiel könnte ich zu Hause sitzen und entscheiden zum Geschäft an der Ecke zu gehen, um Kekse zu kaufen und danach meinen Nachmittags-Tee zu Hause mit Keksen zu genießen. Mein übergeordnetes und bewusstes Ziel ist es also, Kekse zu kaufen, Tee zu kochen und meine Pause zu genießen. Mit diesem Ziel vor Augen ziehe ich meine Schuhe an, stecke mein Portemonnaie ein und mache mich auf den Weg zum Geschäft, wobei ich sicherstelle, dass ich nach beiden Seiten blicke bevor ich die Straße überquere. Im Geschäft wähle ich die Kekse aus, unterhalte mich mit dem Geschäftsinhaber, kaufe meine Kekse, kehre wohlbehalten nach Hause zurück und setze das Wasser auf. Jede dieser Handlungen beruht auf einem eigenen Ziel, doch kaum eines dieser Ziele wurde bewusst getroffen. Doch da solche Ziel-Zustände unser Verhalten auch dann beeinflussen, wenn wir uns ihrer nicht bewusst sind, kann die Zieltheorie Coaches helfen, solche Dynamiken zu erkennen und ihre KlientInnen im Erforschen, Identifizieren und Verändern hinderlicher, impliziter Ziele zu unterstützen, so dass einer sinnhaften, positiven Veränderung weniger im Weg steht. (Für eine umfassende Auseinandersetzung damit, wie Handlungen durch unbewusste Ziele initiiert und motiviert sein können, siehe Custers und Aarts 2010).

\section{Selbst-konkordante Ziele}

Ein/e Coachee engagiert sich in der Regel sehr viel stärker für selbst-kongruente Ziele, die mit seinen/ihren innersten Werten oder mit seiner/ihrer gewünschten Entwicklungsrichtung übereinstimmen. Die Selbstkonkordanz-Theorie
(Sheldon und Elliot 1998) bietet Coaches einen wertvollen Bezugsrahmen, um mit den Beweggründen und Motivationen zu arbeiten, die der Zielwahl und dem Zielstreben zugrunde liegen.

Selbstkonkordanz bezeichnet den Grad, zu dem ein Ziel mit den intrinsischen Interessen, Motivationen und Werten eines Individuums übereinstimmt. Sie zeigt auf einfache und zugleich kraftvolle Weise die Verbindung zwischen Werten und Zielen auf. Abgeleitet von der Selbstbestimmungstheorie (Deci und Ryan 1980), betont das Selbstkonkordanz-Modell das Ausmaß, in dem ein Individuum seine Ziele als die seines eigenen, authentischen Selbst oder als von außen auferlegt erlebt.

Der Selbstkonkordanz-Ansatz beschreibt den wahrgenommenen Ursprung einer Kausalität auf einem Kontinuum zwischen kontrollierten (externalen) Faktoren und internalen (autonomen) Aspekten. Es ist wichtig zu betonen, dass die Wahrnehmung des Individuums bestimmt, in welchem Maß ein Ziel als integriert empfunden wird, und wo es auf dem Kontinuum zwischen internal und external einzuordnen ist. Es ist entscheidend, dass die Ziele eines/ einer Coachee so selbstkongruent wie möglich sind, und es kann sein, dass Coaches im Coaching-Prozess aktiv darauf hinarbeiten müssen, dass die Ziele ihres/ihrer Coachee mit seiner/ihrer Person und seinem/ihrem Kongruenzempfinden in Einklang kommen.

Mindestens vier Faktoren begünstigen eine wirkungsvolle Anpassung in diesem Sinne (Sheldon und Elliot 1999): Erstens muss der/die Coachee überdauernde und authentische Wünsche von vorübergehenden oder oberflächlichen Launen unterscheiden lernen, so dass sein/ihr Bemühen wirkungsvoll ausgerichtet ist. Zweitens muss der/die Coachee erkennen, welche Ziele seine/ihre eigenen Interessen, und welche seiner/ihrer Ziele die Interessen anderer vertreten (Sheldon 2002). Diese Erkenntnisse setzen ein hohes $\mathrm{Maß}$ an Bewusstheit voraus. Da es erhebliche Unterschiede in Individuen im Grad ihrer Bewusstheit über sich selbst gibt (Church 1997), kann dieser Schritt einigen Coachees sehr schwer fallen. Drittens müssen die Ziele inhaltlich so formuliert werden, dass sie mit den internalen Bedürfnissen und Werten des/der Coachee übereinstimmen. Viertens muss der Coach erkennen, wenn ein Ziel nicht selbst-konkordant ist, und das Ziel so umformulieren oder umdeuten können, dass es den Bedürfnissen und Werten des/der Coachee entspricht.

\section{Zielhierarchien}

Zielhierarchien bieten die Möglichkeit, Selbst-Konkordanz $\mathrm{zu}$ operationalisieren und greifbar zu machen, gleichzeitig stellen sie Bezüge zwischen Werten, Zielen und spezifischen Handlungsschritten her. Ziele lassen sich hier- 
archisch ordnen, indem konkrete, spezifische Ziele unter großen, abstrakten Zielen zusammengefasst werden (Chulef et al. 2001), ähnlich der „Big Five“ Persönlichkeitszüge (Costa und McCrae 1992). Entsprechend werden abstrakte Ziele wie „ein/e erfolgreiche/r Unternehmensleiter/in sein“ vertikal höher eingestuft als das spezifische Ziel niederer Ordnung ,den Unternehmensgewinn im nächsten Quartal um $25 \%$ steigern“. Es gibt empirische Evidenz, die diese Auffassung stützt (Chulef et al. 2001; Oishi et al. 1998).

Aus dieser Perspektive lassen sich Ziele höherer Ordnung mit Werten gleichsetzen. Ein Ansatz, wie die Zieltheorie gewinnbringend fürs Coaching eingesetzt werden kann, besteht darin, Werte als höherstehende, abstrakte Ziele zu verstehen, die niederen, spezifischen Zielen übergeordnet sind, welche wiederum über spezifischen Handlungsschritten stehen.

Sich Werte, Ziele und Handlungen in dieser Weise als Teil einer Hierarchie zu veranschaulichen, gibt Coaches einen extrem unterstützenden Bezugsrahmen zum Erarbeiten von Fallkonzepten in ihrer Coaching-Praxis an die Hand (Abb. 2).

Um dieses Modell in der Coaching-Praxis zu verwenden ist es wichtig, soweit wie möglich vertikale und horizontale Kongruenz herzustellen. Vertikale Ausrichtung bedeutet, dass nicht nur die Ziele mit den höchsten Werten der KlientInnen in Einklang stehen, sondern auch alle darauf zuführenden Handlungen. Darüber hinaus ist eine horizontale Ausrichtung wichtig, so dass die Ziele komplementär zueinander sind und sich gegenseitig unterstützen, statt sich, wie zuvor erwähnt, zu konkurrieren oder zu widersprechen. Denn dies würde bedeuten, dass das Verfolgen eines Ziels das Verfolgen eines anderen beeinträchtigt. Natürlich ist eine solche Ausrichtung nicht immer möglich. Trotzdem kann allein das Aufzeigen konkurrierender oder widersprechender Ziele oder einer fehlenden Passung zwischen Zielen und Werten den Coachees neue Einsichten und Perspek-

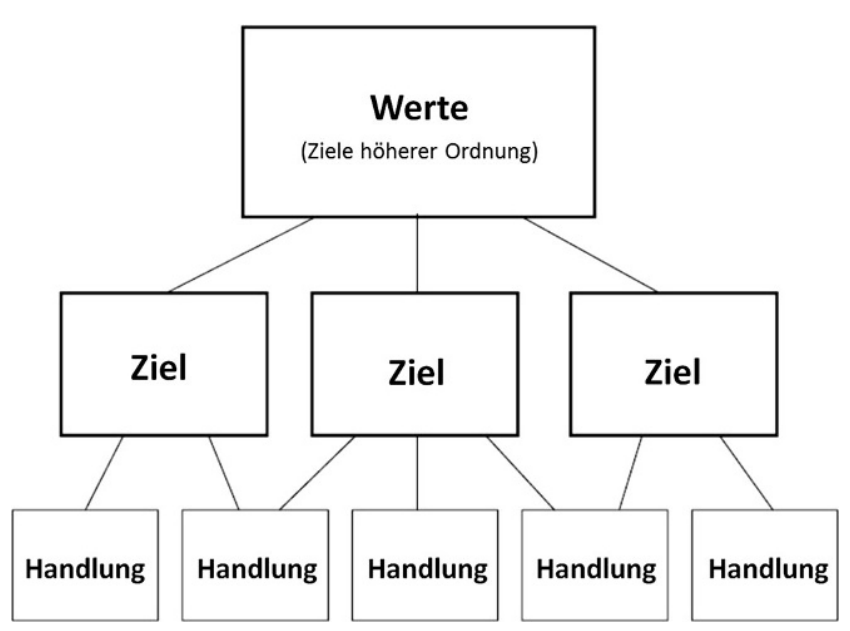

Abb. 2 Zielhierarchie-Chart tiven eröffnen, was wiederum neue Möglichkeiten einlädt, wie der Veränderungsprozess gestaltet werden kann.

\section{Zielvernachlässigung}

Das Hierarchiemodell ist auch deshalb hilfreich für Coaching, weil es die Auswirkung einer Vernachlässigung von Zielen deutlich aufzeigen kann. Zielvernachlässigung wird in der Coaching Literatur eher selten erwähnt, obwohl das Konzept wichtige Konsequenzen für die Coaching-Praxis beinhaltet. Es bezieht sich auf die Missachtung eines Ziels oder einer Aufgabe, obwohl diese als wichtig erkannt wurde (Duncan et al. 1996). Zielvernachlässigung tritt dann auf, wenn wir unsere Aufmerksamkeit nicht einem definierten, wichtigen Ziel schenken, sondern uns stattdessen auf ein anderes Ziel oder eine andere Aufgabe konzentrieren, so dass eine Diskrepanz zwischen den für das ursprüngliche Ziel erforderlichen Handlungen und den tatsächlich ausgeführten Handlungen entsteht.

Menschen sind ihrer Natur nach zielgeleitete Organismen (Deci und Ryan 2002). Alles Verhalten (Verhalten wird hier breit definiert und beinhaltet Gedanken, Gefühle und körperliche Handlungen) erhält seine Form, Richtung, Sinn und Bedeutung durch das Ziel, das wir verfolgen. Dabei wird ein Großteil unseres Verhaltens durch Ziele bestimmt, die außerhalb unserer unmittelbar bewussten Aufmerksamkeit liegen. Wenn wir das Zielhierarchie-Modell heranziehen, so sind es die Ziele höherer Ordnung, die den Zielen und Handlungen niederer Ordnung Richtung, Sinn und Bedeutung geben.

Wenn die Selbstregulation auf den höheren Ebenen einer Zielhierarchie nicht wirken kann (zum Beispiel, weil diesen Werten nicht genügend Aufmerksamkeit geschenkt wird), übernehmen die Ziele tieferer Ebenen und dominieren das nach Außen gezeigte Verhalten (Carver und Scheier 1998). Die Führung des menschlichen Systems stellt sich also auf einer niedrigeren Stufe des Verhaltens ein (regrediert).

Dieses technisch anmutende, psychologische Verständnis hat für die Coaching-Praxis eine wichtige Bedeutung. Dies deshalb, weil die untergeordneten Ziele einer Hierarchie typischerweise im Vergleich zu den höheren Zielen kaum eine Bedeutung haben. Denn bei den untergeordneten Zielen handelt es sich meist um unliebsame Aufgaben, die uns nur darüber schmackhaft werden, dass wir durch ihre Erfüllung unseren höheren Werten folgen.

Wenn wir es verpassen, die höheren Werte unserer Zielhierarchie konsequent im Fokus zu behalten, und uns zu sehr auf das Erreichen niederer Ziele konzentrieren, werden letztere zu unseren übergeordneten und dominanten Werten. Die niederen Ziele an sich sind allerdings oft extrem unbefriedigend. 
Abb. 3 Zielhierarchie-Chart bei Zielvernachlässigung



Im unten dargestellten Beispiel ist das übergeordnete Ziel, ,ein/e herausragende/r Anwalt/Anwältin zu sein“, und viele Studienabgänger dürften mit der Absicht in Rechtsberufe eintreten, herausragende AnwältInnen zu werden und ihren KlientInnen zu Gerechtigkeit zu verhelfen (Abb. 3). Um herausragende AnwältInnen zu werden, müssen sie hart arbeiten, konkrete Beiträge in ihrer Kanzlei leisten und ausreichend Erträge erwirtschaften. Das Erreichen dieser Zwischenziele wiederum wird nur durch Unterziele wie Administration, Rechnungsstellungen und derlei erreicht. Allzu oft legen Individuen zu viel Aufmerksamkeit auf diese niederen Zielebenen (wie Erträge erwirtschaften und Rechnungen schreiben), und verlieren darüber ihre höheren Werte aus dem Blick, was leicht zu Ziel-Unzufriedenheit und Sinnverlust in Bezug auf die eigene Tätigkeit führt.

Dieser Bezugsrahmen kann Coaches und ihren Coachees wertvolle Einsichten in die psychologischen Mechanismen vermitteln, die Ziel-Unzufriedenheit zugrunde liegen, und zur Entwicklung von Werkzeugen und Techniken beitragen, die für KlientInnen in ihrem Coaching-Prozess hilfreich sind. Wenn wir beispielsweise KlientInnen darin unterstützen, ihre Aufmerksamkeit abstrakteren Zielen zuzuwenden, helfen wir ihnen, sich mit ihren höheren Werten zu verbinden. Eventuell redefinieren sie dann auch ihre konkreten Ziele, fühlen sich neu belebt und wieder in einen sinnhaften, positiven Veränderungsprozess eingebunden.

\section{Fazit}

Wie aus diesem kurzen Überblick über einige zentrale Konstrukte ersichtlich wird, hat die Zieltheorie der CoachingPraxis viel zu bieten. Die Frage ist nun, wie die Informatio- nen so geordnet werden können, dass sie für die CoachingPraxis möglichst leicht zugänglich und nützlich sind?

Eine Antwort darauf, wie dieses umfangreiche Wissen gegliedert werden kann, ist das visuelle Modell unter Abb. 4. Es stellt verschiedene Faktoren, die in Bezug zu zielorientiertem Coaching stehen, in ihren Zusammenhängen dar. Aber Achtung: Wie bei allen Modellen zeigt dieses nur einige und keineswegs alle Möglichkeiten, wie die Faktoren im Coaching miteinander interagieren. Es repräsentiert mein eigenes Verständnis und meine persönliche Erfahrung, und ich möchte die LeserInnen ermutigen, die Begrenzungen dieses Modells vor dem Hintergrund der eigenen Coaching-Erfahrung zu erkunden, es anzupassen und $\mathrm{zu}$ erweitern, um daraus einen persönlich passenden Bezugsrahmen zu formen.

Das Modell versucht, Schlüssel-Aspekte zielorientierten Coachings wiederzugeben und unterstreicht gewisse Faktoren, denen im Coaching-Prozess besondere Beachtung zukommt. Der Prozess als Ganzes ist durch bestimmte Bedürfnisse motiviert (im Modell links dargestellt), wofür sowohl individuelle als auch kontextuelle/organisatorische Faktoren eine Rolle spielen. Diese Bedürfnisse beeinflussen das Individuum in der Art seiner Anteilnahme am Zielsetzungsprozess. $\mathrm{Zu}$ den individuellen Faktoren zählen wahrgenommene Defizite und Möglichkeiten, psychologische Bedürfnisse, die Persönlichkeitsstruktur und verfügbare Ressourcen (oder deren Mangel). Beispiele für kontextuelle oder organisatorische Faktoren beinhalten die Komplexität des Systems, die soziale Kultur, die Art der zwischenmenschlichen Beziehungen, Belohnungen und Bestrafungen, und verfügbare Ressourcen (oder deren Mangel).

Der Zielsetzungsprozess verläuft selten geradlinig. Selbst wo Coaching von einer Organisation mit einem bestimmten Ziel vor Augen in Auftrag gegeben wird, kann 


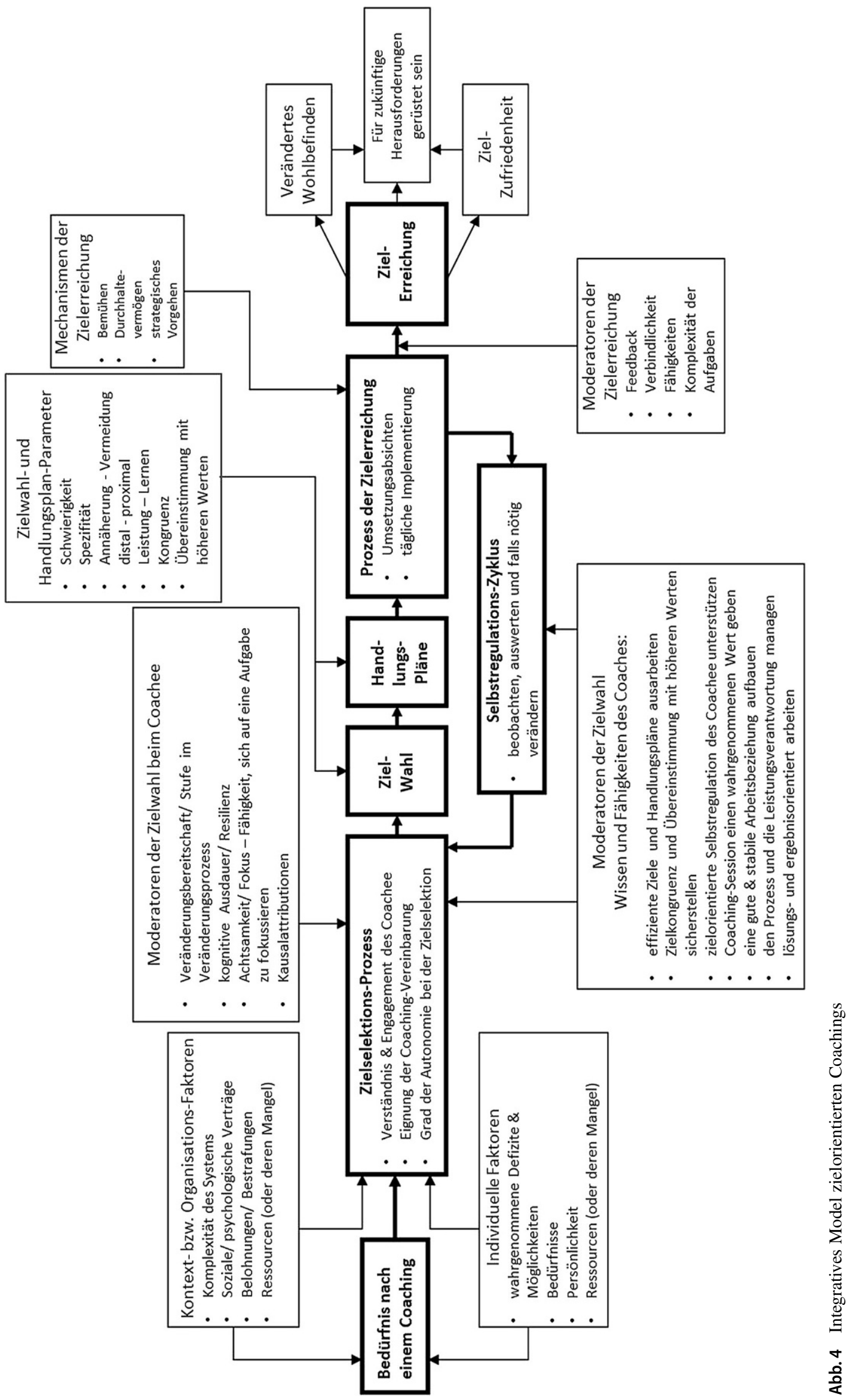


sich der Zielsetzungsprozess verworren und komplex gestalten. Es ist ein häufiger Fallstrick, spezifische Ziele im Coaching-Prozess zu früh und mit Hast festzulegen. Schlüsselthemen und weitgefasste Initialziele müssen zwar schon sehr früh diskutiert werden, um dem Dialog einen Zweck und eine Richtung zu geben, doch der Coach sollte während der Zielwahl eine Reihe von Faktoren berücksichtigen. Ein wichtiger Faktor dabei ist, wie Coachees den CoachingProzess aufnehmen und sich auf diesen einlassen.

Einige Coachees erscheinen zu ihrem ersten CoachingTermin mit wenig Ahnung über Coaching. Die Eignung und Klarheit der Coaching-Vereinbarung (formal oder informal) spielt dann eine ebenso wichtige Rolle für das Mitwirken der Coachees im Zielselektionsprozess wie der Grad an Autonomie bei der Zielwahl.

\section{Zielsetzungs-Moderatoren: Die Eigenschaften der Coachees}

Ein anderer Faktor, der den Zielselektionsprozess beeinflusst, ist die Bereitschaft des/der Coachee, sich zu verändern. Coaches müssen berücksichtigen, ob sich die Coachees in ihren Veränderungsprozessen im Stadium der Absichtslosigkeit, der Vorbereitung oder der Handlung befinden (für eine hilfreiche Referenz für die Anwendung des Transtheoretischen Modells auf eine Bandbreite von Zielen, siehe Prochaska et al. 1994). Das Transtheoretische Modell postuliert, dass Veränderung das Durchlaufen einer Reihe identifizierbarer, sich überlappender Stadien beinhaltet. Fünf dieser Stadien sind direkt für die Zielsetzung im Coaching relevant. Diese Stadien sind:

1. Absichtslosigkeit: Keine Absicht, sich in vorhersehbarer Zeit zu verändern.

2. Absichtsbildung: Erwägung sich zu verändern, ohne etwas umgesetzt zu haben.

3. Vorbereitung: Erhöhte Veränderungsbereitschaft und Absicht, sich in naher Zukunft zu verändern; meist sind bereits erste kleine Schritte erfolgt.

4. Handlung: Das Zeigen neuer Verhaltensweisen seit kurzer Zeit (weniger als 6 Monate)

5. Erhaltung: Dauerhaftes Zeigen des neuen Verhaltens über eine gewisse Zeit (ca. 6 Monate).

\section{Stadium-spezifische Coaching- Strategien}

In der Phase der Absichtslosigkeit geht es vor allem darum, das Bewusstsein der Coachee zu erhöhen und ihnen so viele Informationen wie möglich zugänglich zu machen, so dass sie zum Handeln angeregt werden. Es gibt vielfältige
Möglichkeiten, das Bewusstsein zu erhöhen. Diese umfassen zum Beispiel multi-rater Feedbackbögen, qualitatives Feedback, Verkaufs- oder Leistungszahlen, oder andere, relevante Informationen.

Die Phase der Absichtsbildung ist vorwiegend durch Ambivalenz charakterisiert, dem gleichzeitigen Auftreten von zwei oder mehr in Konflikt stehenden Wünschen, Gefühlen, Überzeugungen oder Meinungen. Individuen in der Phase der Absichtsbildung müssen vor allem darin unterstützt werden, diese Ambivalenz zu erkunden, und sollten nicht gedrängt werden, ein Ziel zu setzen, bevor sie dazu bereit sind. Eine zu frühe oder zu präzise Zielsetzung führt in dieser Phase häufig dazu, dass Coachees sich innerlich vom Zielselektionsprozess zurückziehen.

In der Vorbereitungs-Phase begibt sich der/die Coachee in die Startlöcher für Veränderung. Das Ziel dieser Phase ist, sich Veränderung verbindlich vorzunehmen. Der Coach sollte dem/der Coachee hier helfen, eine klare Vision der Zukunft zu entwerfen (abstrakte Ziele), sowie sich kleine, leicht erreichbare aber konsequente Schritte in dieser Richtung zu setzen. Der Fortschritt sollte in dieser Phase genau beobachtet, und neues, erwünschtes Verhalten verstärkt werden, indem das Erreichen kleiner Unter-Ziele anerkannt und gefeiert wird.

In der Handlungs- und Erhaltungsphase wird auf den vorhergehenden Erfolgen aufgebaut und die selbstgerichtete Veränderung maximiert. Dafür werden mehr herausfordernde Ziele gesetzt und Strategien entworfen, wie die Veränderung über die Zeit erhalten werden kann.

Es ist eine Kunst, Ziele im Coaching wirkungsvoll zu nutzen. Weitere Faktoren, die sich auf die Zielselektion auswirken, sind das Ausmaß, indem der/die Coachee Autonomie und Mitspracherecht bei der Zielselektion empfindet, sowie seine/ihre Fähigkeit, sich anstehenden Aufgaben anzunehmen und auf Widrigkeiten flexibel zu reagieren.

\section{Moderatoren in der Coaching-Sitzung}

Auch in der Coaching-Sitzung selbst gibt es eine Reihe Faktoren, die den Zielselektionsprozess beeinflussen. Dazu zählen die Fähigkeiten des Coaches, effektive Ziele zu setzen und Handlungsplanung zu ermöglichen, wie auch die zielorientierte Selbstregulation des/der Coachee so zu unterstützen, dass maximale Zielkongruenz resultiert.

Der Erfolg des genannten ist auch davon abhängig, ob der Coach der Sitzung einen wahrgenommenen Wert verleihen und eine starke Allianz mit dem/der Coachee aufbauen kann (Gray 2007). Alles theoretische Wissen über die Zieltheorie führt zu nichts, wenn der Coach es nicht umsetzen kann, indem er den Prozess des Zielstrebens lenkt, den/die Coachee in die Verantwortung nimmt und lösungs- und ergebnisorientiert arbeitet. 


\section{Zielwahl und Handlungsplanung}

Zielwahl und Handlungsplanung sind Ergebnisse des Zielselektionsprozesses. Obwohl das Modell sie als lineare Prozesse darstellt, ist es ist wichtig zu betonen, dass sie sich in Wirklichkeit iterativ gestalten, mit einem Vor und $\mathrm{Zu}$ rück zwischen den einzelnen Phasen. Parameter der Zielwahl und Handlungsplanung beinhalten Zielschwierigkeit und -spezifität, ob es Annäherungs- oder Vermeidungsziele sind, den Zeitrahmen (distal oder proximal), und die Leistungs- oder Lernorientierung.

Zielwahl ist ein notwendiger, aber nicht hinreichender Teil des Coaching-Prozesses; es müssen auch Handlungspläne geschmiedet und ausgeführt werden. In der Handlungsplanung wird ein systematischer Weg zum Erreichen der Ziele entwickelt. Dies ist insbesondere für Individuen wichtig, die über geringe Selbstregulationsfähigkeiten verfügen (Kirschenbaum, Humphrey und Malett 1981). Die Rolle des Coaches besteht darin, den/die Coachee beim Erstellen eines realistischen, durchführbaren Handlungsplans und dazu passenden Strategien zu unterstützen, so dass sein/ihr Zielstreben, sein/ihr Leistungs- und Durchhaltewille angespornt werden und auch bei auftretenden Schwierigkeiten Bestand haben können.

Ein Schlüssel erfolgreicher Handlungsplanung ist es, den Übergang eines/einer Coachee von einer abwägenden zu einer umsetzenden Haltung zu begleiten (Gollwitzer 1999; Heckhausen und Gollwitzer 1987). In der abwägenden Phase bedenkt der/die Coachee die Vor- und Nachteile von Handlungen sorgfältig und schenkt konkurrierenden Zielen oder Handlungsplänen genaue Aufmerksamkeit (Carver und Scheier 1998). Sobald die Entscheidung zu handeln getroffen wurde, ergibt sich eine Haltung, die auf Umsetzung ausgerichtet ist. Diese Haltung hat eine entschlossene und fokussierte Qualität, und hat die Tendenz stärker an Erfolg als an Misserfolg zu denken - ein wichtiger Punkt fürs Coaching.

Der Schritt von der abwägenden zur umsetzenden Haltung ist auch deshalb wichtig, weil Individuen in der Umsetzungsphase verstärkt daran glauben, dass sie Einfluss auf die Ergebnisse ihres Tuns haben (Gollwitzer und Kinney 1989), und die Wahrscheinlichkeit ihres Erfolgs positiv und optimistisch beurteilen (Taylor und Gollwitzer 1995). Diese Faktoren werden mit höherer Selbstwirksamkeit, Selbstregulation und Zielerreichung in Verbindung gebracht (Bandura 1982).

\section{Selbstregulations-Zyklus, Feedback und Zielzufriedenheit}

Handlungen $\mathrm{zu}$ beobachten, zu evaluieren und dem/der Coachee Feedback zu geben, während er/sie den Selbst-
regulations-Zyklus durchläuft, sind wichtige Elemente des Coaching-Prozesses. Allerdings verfügen nicht alle Menschen über eine hohe Selbstreflexion (Jordan und Troth 2002), so dass ein Coach unter Umständen Handlungspläne finden muss, die sich auf beobachtbares und leicht $\mathrm{zu}$ erhebendes Verhalten konzentrieren.

Was erfasst wird, hängt natürlich von Ziel und Situation des/der Coachee ab. Manche Verhaltensweisen lassen sich leichter erheben als andere. Fitnessübungen und aktivitätsbasierte Handlungen lassen sich sehr direkt erfassen. Zwischenmenschliche Kompetenzen und Kommunikationsmuster am Arbeitsplatz sind schwieriger zu erfassen, und Coach und Coachee müssen für deren Erhebung und Evaluation kreativer sein.

In weiser Voraussicht sollten Ziele gewählt werden, die hilfreiches Feedback ermöglichen, da das richtige Feedback entscheidende Informationen darüber liefert, ob und wie Folgeziele und dazugehörige Handlungen modifiziert werden müssen. Wenn der Prozess gut gemacht wird, mündet er in erfolgreiches Erreichen des Ziels. Ziele, die in Übereinstimmung mit den inneren Werten des/der Coachee gebracht worden waren, rufen bei Erreichen Zufriedenheit hervor. Die damit verbundenen positiven Gefühle können eine wichtige Rolle darin spielen, Coachees für ihr Engagement bei zukünftigen Herausforderungen zu primen.

\section{Ist die Zieltheorie für die Praxis jetzt also relevant oder nicht?}

Obwohl aus obiger Diskussion hervorgeht, dass die Zieltheorie Informationen darüber liefern kann, was in Coaching-Sitzungen passiert, und auch eine große Relevanz für den gesamten Coaching-Prozess hat, stellt sich die Frage: Hat die Zieltheorie tatsächlich für die Praxis Relevanz? Steht die Fähigkeit eines Coaches, zielorientiert zu arbeiten, in Verbindung mit dem Coaching-Ergebnis? Diese Frage ist zentral für die Weiterentwicklung empirisch basierter Coaching-Praxis.

In der psychotherapeutischen Literatur weisen zahlreiche Forschungsarbeiten überzeugend nach, dass die wichtigsten Faktoren für das therapeutische Ergebnis die sogenannten „common factors“ sind - die Fähigkeit der TherapeutInnen, eine Arbeitsbeziehung mit KlientInnen aufzubauen, die Vertrauen, Wärme und Respekt für die Autonomie der KlientInnen beinhaltet (Lampropoulos 2000). Es überrascht nicht, dass in der Coaching-Literatur oft angenommen wird, dass es sich im Coaching ähnlich verhält (McKenna und Davis 2009a). Coaching ist jedoch nicht Therapie. Die Ziele von Coaching und Therapie sind unterschiedlich.

Bisher gab es wenig Studien, die die Bedeutung von Zielen in der Coaching-Beziehung untersucht haben, und es interessierte mich, welcher Aspekt im Coaching stärker mit 
Ergebnissen im Coaching korrelieren würde - ein zielorientierter Coaching-Ansatz, oder die sogenannten ,common factors" des personenzentrierten Ansatzes (Grant 2013a). Um dies zu untersuchen, entwarf ich eine within-subjects (pre-post) Coaching-Studie, in der 49 erwachsene Coachees (12 Männer, 37 Frauen, Alter $M=37,5$ Jahre) sich persönliche Ziele vornahmen und ein 10-12 wöchiges, lösungsorientiertes, kognitiv-behaviorales und persönliches Coaching-Programm mit 5 Sitzungen nach dem GROW Modell $^{1}$ (Whitmore 1992) durchliefen.

Die TeilnehmerInnen wurden gebeten, ihr aus der Coaching-Beziehung resultierendes, erwünschtes Ergebnis zu benennen (z. B. ihr Ziel), und es dann auf einer Skala von $0 \%$ (nichts erreicht) bis $100 \%$ (vollständiges Erreichen) zu beurteilen. Die psychologische Gesundheit wurde mithilfe der Depressions-Angst-Stress-Skalen (DASS-21, Lovibond und Lovibond 1995) und einer 18-Item-Version der Ryff's Psychological Wellbeing Scales (Ryff und Keyes 1995) erfasst. Zusätzlich wurde Selbst-Einsicht über die Einsichtsdimension des Self-reflection and Insight Scale (SRIS; Grant et al. 2002) erhoben.

Um zu sehen, welcher Aspekt der Coaching-Beziehung der bessere Prädiktor für Erfolg war, wurden zwei Messinstrumente zum Erfassen der Coaching-Beziehung verwendet. Der zielorientierte Aspekt wurde mit einer angepassten Version des Goal-focused Coaching Skills Questionnnaire (GCSQ; Grant und Cavanagh 2007) gemessen. Items dieser Skala beinhalten zum Beispiel: „Mein Coach half mir, einfache, klare und erreichbare Handlungspläne zu entwickeln.“; „Wenn ich vereinbarte Umsetzungsschritte nicht ausführte, besprachen wir dies gemeinsam.“; „Die im Coaching vereinbarten Ziele waren mir wichtig.“; „Mein Coach hat mich nach meinem Zielfortschritt gefragt.“; „Die gesetzten Ziele waren herausfordernd, aber erreichbar.“

Der „common factors“ Aspekt wurde mit einer angepassten Version von Deci und Ryans (2005) Perceived Autonomy Support Scale (PASS) erhoben. Items dieser Skala beinhalten zum Beispiel: „Mein Coach fragte mich, wie ich gerne vorgehen würde.“; „Ich spüre, dass mein Coach mich mag.“; „Mein Coach ermutigte mich, Fragen zu stellen.“; „Ich spüre, dass mein Coach mich schätzt.“; „Ich fühlte mich von meinem Coach verstanden.“; „Ich habe viel Vertrauen in meinen Coach.“

\footnotetext{
${ }^{1}$ Das GROW Modell wird häufig genutzt, um Coaching-Gespräche zu strukturieren. Es wird ein Ziel für die Sitzung festgelegt, dann die reale Situation betrachtet, mögliche Optionen diskutiert, und schließlich die Sitzung mit der Entscheidung für einige konkrete Handlungsschritte beschlossen. Dies mag wie ein simpler, linearer Prozess erscheinen, das GROW Modell kann jedoch differenziert und iterativ angewandt werden, indem das Gespräch sich in den Phasen vor und zurückbewegt. Für weitere Informationen über die Anwendung des GROW Modells, siehe Grant (2011).
}

Das Coaching-Programm schien den Klientinnen effektiv und erfolgreich beim Erreichen ihrer durch die Coaching-Beziehung erwünschten Ergebnisse zu helfen: Es gab nach dem Coaching einen signifikanten Anstieg bei der Zielerreichung $\left(t_{1,48}(11,43) ; p<0,001\right)$ und der Einsicht $\left(t_{1,48}(2,61) ; p<0,05\right)$, sowie eine signifikante Abnahme bei Ängstlichkeit $\left(t_{1,48}(2,89) ; p<0,001\right)$ und Stress $\left(t_{1,48}(2,13)\right.$; $p<0,05)$. Bei Depression oder psychologischem Wohlbefinden wurden keine Unterschiede verzeichnet.

Das Hauptinteresse galt jedoch dem Zusammenhang zwischen den durch das Coaching erreichten Erfolgen und den verschiedenen Aspekten des verwendeten CoachingAnsatzes. Es gab eine signifikante Korrelation zwischen dem Coaching-Erfolg, definiert über das Maß, in dem KlientInnen ihre erwünschten Ergebnisse erreicht hatten (z. B. Zielerreichung), und dem GCSQ $(r=0,43 ; p<0,01)$. Ebenso war die Korrelation zwischen Coaching-Erfolg (definiert über das Maß, in dem KlientInnen ihre erwünschten Ergebnisse erreicht hatten) und dem PASS $(r=0,29 ; p<0,05)$ signifikant. Es überrascht nicht, dass auch eine signifikante Korrelation zwischen dem GCSQ und dem PASS bestand $(r=0,61 ; p<0,001)$. Dies scheint darauf hinzudeuten, dass sowohl ein zielorientierter Coaching-Ansatz als auch ein personenzentrierter ,,common factors“ Coaching-Ansatz zu Erfolg durch Coaching beitragen.

Die zentrale Erkenntnis besteht darin, dass die Korrelation zwischen Coaching-Erfolg (Zielerreichung) und dem zielorientierten Coaching-Ansatz, der durch den GCSR gemessen wurde, auch dann signifikant blieb, wenn auf den personenzentrierten „common factors“-Ansatz, gemessen durch den PASS, kontrolliert wurde $(r=0,31 ; p=0,05)$. Es gilt festzuhalten, dass wenn der zielorientierte Ansatz, gemessen durch den GCSY, kontrolliert wurde, der Zusammenhang zwischen Coaching-Erfolg (Zielerreichung) und dem PASS nicht signifikant war $(r=0,03 ; p=0,81)$.

Diese Befunde lassen vermuten, dass der Verwendung von Zielen im Coaching in der Tat praktische Bedeutung zukommt, insofern als sich im Coaching-Kontext ein zielorientierter Coaching-Ansatz effektiver zeigt als ein personenzentrierter „common factors“-Ansatz. Dies besagt keineswegs, dass eine personenzentrierte Beziehung nicht bedeutsam ist. Es erinnert vielmehr daran, dass sich die Beziehung im Coaching von der Beziehung in Beratung oder Therapie unterscheidet, und dass Coaches sich dessen bewusst sein sollten, dass sie von ihren KlientInnen bezahlt werden, um diesen bei zweckgerichteten, positiven Veränderungen in ihrem privaten und professionellen Leben behilflich zu sein. 


\section{Zusammenfassung}

Coaches mögen ihre Rolle als Unterstützende verstehen, die ihren KlientInnen beim Erkunden ihrer Werte und beim Klären ihrer Absichten helfen, und mit ihnen am Erreichen ihrer Vorhaben arbeiten. Dafür lassen sich Metaphern finden wie „die persönliche Geschichte neu schreiben“, „den Kompass neu ausrichten“ oder ,sich sicher durch die Wirren des Lebens bewegen“. Trotz unterschiedlicher Semantik lässt sich jedoch sagen, dass Coaching notwendigerweise ein zielorientiertes Unterfangen ist.

Der in diesem Artikel vorgestellte integrative und zielorientierte Coaching-Ansatz stellt eine vielseitige Methodologie dar, um Individuen und Organisationen bei Veränderungsprozessen nachhaltig zu unterstützen. Der Schlüssel liegt in einer sachkundigen und flexiblen Zielsetzung durch den Coach. Das Verständnis verschiedener Zielarten und ihrer Bedeutung für Veränderung, sowie das Begleiten von Zielfindungsprozessen, ermöglicht professionellen Coaches effektiv mit ihren KlientInnen an Einsichten und Verhaltensveränderungen zu arbeiten, die ihre Arbeitsleistung, ihr Arbeitserleben, sowie - und dies ist der wichtigste Punkt - ihr persönliches Wohlbefinden und Selbstverständnis erhöht.

Open Access Dieser Artikel wird unter der Creative Commons Namensnennung 4.0 International Lizenz (http://creativecommons.org/ licenses/by/4.0/deed.de) veröffentlicht, welche die Nutzung, Vervielfältigung, Bearbeitung, Verbreitung und Wiedergabe in jeglichem Medium und Format erlaubt, sofern Sie den/die ursprünglichen Autor(en) und die Quelle ordnungsgemäß nennen, einen Link zur Creative Commons Lizenz beifügen und angeben, ob Änderungen vorgenommen wurden.

\section{Literatur}

Association of Coaching (2012). Definition of coaching. http://www. associationforcoaching.com/about/about03.htm. Zugegriffen: 19. Apr. 2012.

Austin, J. T., \& Vancouver, J.B. (1996). Goal constructs in psychology: structure, process, and content. Psychological Bulletin, 120(3), $338-375$

Bandura, A. (1982). Self-efficacy mechanism in human agency. American Psychologist, 37, 122-147.

Bargh, J. A., Gollwitzer, P. M., Lee-Chai, A., Barndollar, K., \& Trötschel, R. (2001). The automated will: nonconscious activation and pursuit of behavioral goals. Journal of Personality and Social Psychology, 81(6), 1014-1027.

Benavides, L. (2009). The relationship between executive coaching and organizational performance of female executives as a predictor for organizational success. San Fransisco: University of San Fransisco.

Bono, J.E., Purvanova, R. K., Towler, A. J., \& Peterson, D. B. (2009). A survey of executive coaching practices. Personnel Psychology, 62(2), 361-404.

Burke, D., \& Linley, P. (2007). Enhancing goal self-concordance through coaching. International Coaching Psychology Review, 2(1), 62-69.

Carver, C. S., \& Scheier, M.F. (1998). On the self-regulation of behavior. Cambridge: Cambridge University Press.
Chulef, A. S., Read, S. J., \& Walsh, D. A. (2001). A hierarchical taxonomy of human goals. Motivation \& Emotion, 25(3), 191-232.

Church, A.H. (1997). Managerial self-awareness in high-performing individuals in organizations. Journal of Applied Psychology, 82(2), 281-292.

Clutterbuck, D. (2008). What's happening in coaching and mentoring? And what is the difference between them? Development and Learning in Organizations, 22(4), 8-10.

Clutterbuck, D. (2010). Coaching reflection: the liberated coach. Coaching, 3(1), 73-81.

Coats, E. J., Janoff-Bulman, R., \& Alpert, N. (1996). Approach versus avoidance goals: differences in self-evaluation and well-being. Personality and Social Psychology Bulletin, 22(10), 1057-1067.

Cochran, W., \& Tesser, A. (1996). The "What the Hell" effect: some effects of goal proximity and goal framing on performance. In L. Martin \& A. Tesser (Hrsg.), Striving and Feeling (S. 99-123). New Jersey: LEA.

Costa, P.T., \& McCrae, R.R. (1992). Revised neo personality inventory and neo five-factor inventory: professional manual. Odessa: Psychological Assessment Resources.

Cowan, K. (2013). What are the experiences of external executive coaches working with coachees' assigned goals? International Journal of Evidence Based Coaching and Mentoring, 7, 14-25.

Custers, R., \& Aarts, H. (2010). The unconscious will: how the pursuit of goals operates outside of conscious awareness. Science, 329(5987), 47

Deci, E.L., \& Ryan, R. M. (1980). Self-determination theory: when mind mediates behavior. Journal of Mind \& Behavior, 1(1), 33-43.

Deci, E.L., \& Ryan, R. M. (Hrsg.). (2002). Handbook of self-determination research. Rochester: University of Rochester Press.

Deci, E. L., \& Ryan, R. M. (2005). Perceived autonomy support scale. http://www.psych.rochester.edu/SDT/measures/needs_scl.html. Zugegriffen: 5. Aug. 2006.

Dewck, C.S. (1986). Motivational processes affecting learning. American Psychologist, 41(10), 1040-1048.

Doran, G. T. (1981). There's a S.M.A.R.T. way to write management's goals and objectives. Management Review, 70(11), 35-36.

Duncan, J., Emslie, H., Williams, P., Johnson, R., \& Freer, C. (1996). Intelligence and the frontal lobe: the organization of goal-directed behavior. Cognitive Psychology, 30(3), 257-303.

Elliot, A.J., \& McGregor, H.A. (2001). A 2 X 2 achievement goal framework. Journal of Personality and Social Psychology, 80(3), $501-519$.

Elliot, A. J., Sheldon, K. M., \& Church, M. A. (1997). Avoidance personal goals and subjective well-being. Personality \& Social Psychology Bulletin, 23(9), 915-927.

EMCC (2011). European mentoring and coaching council code of conduct for coaching and Mentoring. http://www.emccouncil.org/src/ ultimo/models/Download/4.pdf. Zugegriffen: 19. Apr. 2012.

Emmons, R.A. (1992). Abstract versus concrete goals: personalstriving level, physical illness and psychological wellbeing. Journal of Personality and Social Psychology, 62, 292-300.

Freedman, A.M., \& Perry, J.A. (2010). Executive consulting under pressure: a case study. Consulting Psychology Journal: Practice and Research, 62(3), 189-202.

Gollwitzer, P. M. (1999). Implementation intentions: simple effects of simple plans. American Psychologist, 54(7), 493-503.

Gollwitzer, P. M., \& Kinney, R. F. (1989). Eftects of deliberative and implemental mind-sets on illusion of control. Jouirnal of Per- sonality and Social Psychology, 56, 531-542.

Grant, A. M. (2006). An integrative goal-focused approach to executive coaching. In D. Stober \& A. M. Grant (Hrsg.), Evidence based coaching handbook (S. 153-192). New York: Wiley.

Grant, A. M. (2011). Is it time to regrow the grow model? Issues related to teaching coaching session structures. The Coaching Psychologist, 7(2), 118-126. 
Grant, A. M. (2013a). Autonomy support, relationship satisfaction and goal focus in the coach-coachee relationship: which best predicts coaching success? Coaching, 7(1), 18-38.

Grant, A.M. (2013b). New perspectives on goal setting in coaching practice: an integrated model of goal-focused coaching. In D. Clutterbuck, D. Megginson \& S. David (Hrsg.), Beyond goals: effective strategies for coaching and mentoring (S. 55-84). London: Gower Publishing.

Grant, A. M., \& Cavanagh, M. (2007). The goal-focused coaching skill questionnaire: preliminarily findings. Social Behavior and Personality, 35(6), 751-760.

Grant, A. M., \& Green, J. (2004). Coach yourself. London: Pearson.

Grant, A. M., Franklin, J., \& Langford, P. (2002). The self-reflection and insight scale: a new measure of private self-consciousness. Social Behavior and Personality, 30(8), 821-836.

Grant, A. M., Curtayne, L., \& Burton, G. (2009). Executive coaching enhances goal attainment, resilience and Workplace well-being: a randomised controlled study. The Journal of Positive Psychology, 4(5), 396-407.

Grant, A. M. (2002). Towards a Psychology of Coaching: The Impact of Coaching on Metacognition, Mental Health and Goal Attainment. Dissertation Abstracts International Section A: Humanities and Social Sciences, 63/12, pp. 6094 (June).

Gray, D.E. (2007). Towards a systemic model of coaching supervision: some lessons from psychotherapeutic and counselling models. Australian Psychologist, 42(4), 300-309.

Gregory, J.B., \& Levy, P.E. (2015). How feedback and goals drive behavior: control theory. Washington: American Psychological Association.

Gregory, J. B., Beck, J.W., \& Carr, A.E. (2011). Goals, feedback, and self-regulation: control theory as a natural framework for executive coaching. Consulting Psychology Journal: Practice and Research, 63(1), 26.

Gresham, F. M., Evans, S., \& Elliott, S. N. (1988). Academic and social self-efficacy scale: development and initial validation. Journal of Psychoeducational Assessment, 6(2), 125-138.

Heckhausen, H. \& Gollwitzer, P. M. (1987). Thought contents and cognitive functioning in motivational versus volitional states of mind. Motivation and Emotion, 11, 101-120

Higgins, E. T. (1987). Self-discrepancy: a theory relating self and affect. Psychological Review, 94, 319-340.

Hudson, F. M. (1999). The handbook of coaching. San Francisco: Jossey-Bass.

ICF (2012). International Coach Federation Code of Ethics. http:// www.coachfederation.org/ethics/. Zugegriffen: 19. Apr. 2012.

Jordan, P. J., \& Troth, A.C. (2002). Emotional intelligence and conflict resolution: implications for human resource development. Advances in developing human. Resources, 4(1), 62-79.

Karniol, R., \& Ross, M. (1996). The motivational impact of temporal focus: thinking about the future and the past. Annual Review of Psychology, 47, 593-620.

Kirschenbaum, D. S., Humphrey, L. L., \& Malett, S. D. (1981). Specificity of planning in adult self-control: An applied investigation. Journal of Personality and Social Psychology, 40(5), 941-950. https://doi.org/10.1037/0022-3514.40.5.941.

Klinger, E. (1975). Consequences of commitment to and disengagement from incentives. Psychological Review, 82, 1-25.

Kristof-Brown, A. L., \& Stevens, C. K. (2001). Goal congruence in project teams: does the fit between members' personal mastery and performance goals matter? Journal of Applied Psychology, 86(6), 1083-1095.

Lampropoulos, G. K. (2000). Definitional and research issues in the common factors approach to psychotherapy integration: misconceptions, clarifications, and proposals. Journal of Psychotherapy Integration, 10(4), 415-438.

Lewis-Duarte, M. (2010). Executive Coaching: A Study of Coaches' Use of Influence Tactics. Lewis-Duarte, Melissa: The Claremont Graduate U, US.
Linnenbrink, E. A., Ryan, A. M., \& Pintrich, P.R. (1999). The role of goals and affect in working memory functioning. Learning \& Individual Differences, 11(2), 213-230.

Little, B.R. (1993). Personal projects: a rationale and method for investigation. Environment and Behavior, 15, 273-309.

Locke, E. (2000). Motivation, cognition, and action: an analysis of studies of task goals and knowledge. Applied Psychology, 49(3), 408-429.

Locke, E. A. (1996). Motivation through conscious goal setting. Applied \& Preventive Psychology, 5(2), 117-124.

Locke, E. A., \& Latham, G.P. (2002). Building a practically useful theory of goal setting and task motivation. American Psychologist, 57(9), 705-717.

Lovibond, S.H. \& Lovibond, P.F. (1995). Manual for the Depression Anxiety Stress Scales. (2nd. Ed.) Sydney: Psychology Foundation.

Manderlink, G., \& Harackiewicz, J. M. (1984). Proximal versus distal goal setting and intrinsic motivation. Journal of Personality \& Social Psychology, 47(4), 918-928.

McKenna, D., \& Davis, S. L. (2009a). Hidden in plain sight: the active ingredients of executive coaching. Industrial and organizational psychology. Perspectives on Science and Practice, 2(3), 244-260.

McKenna, D., \& Davis, S.L. (2009b). What is the active ingredients equation for success in executive coaching? Industrial and organizational psychology. Perspectives on Science and Practice, 2(3), 297-304.

Midgley, C., Kaplan, A., \& Middleton, M. (2001). Performance-approach goals: good for what, for whom, under what circumstances, and at what cost? Journal of Educational Psychology, 93(1), $77-86$.

Milare, S. A., \& Yoshida, E. M.P. (2009). Brief intervention in organizations: change in executive coaching. Psicologia em Estudo, 14(4), 717-727.

Moskowitz, G.B., \& Grant, H. (Hrsg.). (2009). The psychology of goals. New York: Guilford.

Oishi, S., Schimmack, U., Diener, E., \& Suh, E. M. (1998). The measurement of values and individualism-collectivism. Personality \& Social Psychology Bulletin, 24(11), 1177-1189.

Ordóñez, L.D., Schweitzer, M.E., Galinsky, A.D., \& Bazerman, M.H (2009). Goals gone wild: the systematic side effects of over-prescribing goal setting. Academy of Management Perspectives, 1, 6-16. https://doi.org/10.5465/AMP.2009.37007999.

Pervin, L.A. (1982). The stasis and flow of behavior: toward a theory of goals

Peterson, D. B. (2011). Good to great coaching. In G. Hernez-Broome \& L. A. Boyce (Hrsg.), Advancing executive coaching: setting the course of successful leadership coaching (S. 83-102). San Francisco: Jossey-Bass.

Prochaska, J. O., Norcross, J.C., \& DiClemente, C. C. (1994). Changing for good. New York: Avon Books.

Raia, A.P. (1965). Goal setting and self-control: an empirical study. Journal of Management Studies, 2(1), 34-53.

Rawsthorne, L. J., \& Elliott, A. J. (1999). Achievement goals and intrinsic motivation: a meta-analytic review. Personality \& Social Psychology Review, 3(4), 326-344.

Ryff, C.D., \& Keyes, C.L.M. (1995). The structure of psychological well-being revisited. Journal of Personality and Social Psychology, 69(4), 719-727.

Sarrazin, P., Vallerand, R., Guillet, E., Pelletier, L., \& Cury, F. (2002). Motivation and dropout in female handballers: a 21-month prospective study. European Journal of Social Psychology, 32(3), $395-418$.

Schnell, E. R. (2005). A case study of executive coaching as a support mechanism during organizational growth and evolution. Consulting Psychology Journal: Practice and Research, 57(1), 41-56.

Seijts, G. H., \& Latham, G.P. (2001). The effect of distal learning, outcome, and proximal goals on a moderately complex task. Journal of Organizational Behavior, 22(3), 291-307. 
Sheldon, K. M. (2002). The self-concordance model of healthy goal striving: when personal goals correctly represent the person. In E. L. Deci \& R. M. Ryan (Hrsg.), Handbook of self-determination reserach (S. 65-86). Rochester: University of Rochester Press.

Sheldon, K., \& Elliot, A. J. (1999). Goal striving, need satisfaction and longitudinal well-being: the self-concordance model. Journal of Personality and Social Psychology, 76(3), 482-497.

Sheldon, K. M., \& Elliot, A. J. (1998). Not all personal goals are personal: comparing autonomous and controlled reasons for goals as predictors of effort and attainment. Personality \& Social Psychology Bulletin, 24(5), 546-557.

Sheldon, K. M., \& Kasser, T. (1995). Coherence and congruence: two aspects of personality integration. Journal of Personality \& Social Psychology, 68(3), 531-543.

Smither, J. W., London, M., Flautt, R., Vargas, Y., \& Kucine, I. (2003). Can working with an executive coach improve multisource feedback ratings over time? A quasi-experimental field study. Personnel Psychology, 56(1), 23-44.

Spence, G. B. (2007). Gas powered coaching: goal attainment scaling and its use in coaching research and practice. International Coaching Psychology Review, 2(2), 155-167.

Stern, L.R. (2009). Challenging some basic assumptions about psychology and executive coaching: who knows best, who is the client, and what are the goals of executive coaching? Industrial and organizational psychology. Perspectives on Science and Practice, 2(3), 268-271.

Street, H. (2002). Exploring relationships between goal setting, goal pursuit and depression: a review. Australian Psychologist, 37(2), 95-103.

Taylor, S. E., Gollwitzer, P. M. (1995). The effects of mindsets on positive illusions. Journal of Personality and Social Psychology, 69, 213-226.

Turner, C. A. (2004). Executive Coaching: The Perception of Executive Coaching from the Executive's Perspective. Turner, Carol Ann: Pepperdine U, US.

WABC (2012). Worldwide assoication of business coaches definition of business coaching. http://www.wabccoaches.com/includes/ popups/definition.html. Zugegriffen: 19. Apr. 2012.

Warshaw, P. R., Davis, F. D. (1985). The accuracy of behavioral intention versus behavioral expectation for predicting behavioral goals. The Journal of Psychology, 119(6), 599-602.

Weldon, E., Yun, S. (2000). The effects of proximal and distal goals on goal level, strategy development, and group performance. Journal of Applied Behavioral Science, 36(3), 336-344.

Whitmore, J. (1992). Coaching for performance. London: Nicholas Brealey.

Winters, D., Latham, G.P. (1996). The effect of learning versus outcome goals on a simple versus a complex task. Group Organization Management, 21(2), 236-250. 